\title{
SALUD ADOLESCENTE EN EL CHACO. NECESIDADES, ACTORES Y POLIIIICAS'
}

\section{ADOLESCENT HEALIH IN CHACO. NEEDS, ACTORS AND POLICIES}

Daniel Maciera ${ }^{2}$, Damian Navarro ${ }^{3}$, Liliana Ensisa ${ }^{4}$, Natalia Espinola ${ }^{5}$, Gabriela Obregón ${ }^{6}$

RESUMEN La adolescencia, edad de bajo riesgo clínico, se asocia con una etapa de formación intelectual e incorporación de hábitos que afectan otras etapas de la vida. En la Argentina, los avances de derechos buscan su correlato en cobertura de necesidades de salud. La interacción entre los sistemas de salud y educación y el papel jugado por la familia y el entorno tienen su reflejo en la carga de adicciones, tabaquismo, consumo de alcohol, conductas sexuales irresponsables y violencia externa y auto-infringida. El trabajo usa técnicas cuanti-cualitativas para analizar el caso chaqueño. El abordaje involucra un mapeo tradicional de trece actores y una encuesta de elección múltiple a más de ochocientos/as jóvenes en una docena de escuelas en los departamentos de San Fernando, Comandante Fernández, General Güemes, Libertador General San Martin y Mayor Fontana. La percepción de organizaciones de la sociedad civil sobre la salud adolescente permite una aproximación a jóvenes no escolarizadas/os. Los resultados revelan la necesidad de coordinación, a fin de reducir la brecha existente entre la necesidad percibida del/la adolescente y la interpretación del adulto, y entre la norma y su traducción en un sistema de cuidados que aún no logró alternativas de acción novedosas ante un fenómeno de incidencia creciente.

Palabras clave: adolescente, comportamiento, políticas públicas.

1 Recibido el 21 de junio de 2018. Aceptado el 22 de febrero de 2019.

${ }^{2}$ Ph.D. en Economía, Boston University. Investigador Independiente CONICET, Investigador Titular Centro de Estudios de Estado y Sociedad (CEDES), y Profesor Titular, Facultad de Ciencias Económicas, Universidad Nacional de Buenos Aires. Mail: danielmaceira@cedes.org.

${ }^{3}$ Profesor en Filosofía. Doctorando en Ciencias Sociales. Becario del CONICET. Docente de la Universidad Nacional del Nordeste. Mail: damian_navarro@hotmail.com.

${ }^{4}$ Médica Pediatra. Coordinadora del Programa Provincial de Salud Integral en la Adolescencia. Ministerio de Salud Pública de la Provincia del Chaco.

Mail: lilianaensisa@hotmail.com.

\footnotetext{
${ }^{5}$ Magíster en Economía. Profesora de la Universidad Nacional de La Plata y Universidad de Buenos Aires. Mail: nespinola12@gmail.com.
}

${ }^{6}$ Estudiante avanzada del Profesorado y la Licenciatura en Filosofía de la Universidad Nacional del Nordeste. Mail: gabriela_obregon@yahoo.com.ar.

\section{INTRODUCCIÓN}

El objetivo del presente artículo es poner en evidencia las comunicaciones y tensiones existentes en la relación demandaaccesibilidad al derecho a la salud de la población adolescente, analizando la realidad de la provincia del Chaco.

La distinción de la adolescencia como una etapa del desarrollo humano con características especificas a ser consideradas en una agenda de desarrollo social está firmemente aceptada. Sin embargo, la población que la conforma no siempre es objeto de políticas públicas de salud específicas (Garay et al., 2015), y prueba de ello es la ausencia - justificada o no, según las perspectivas a ser discutidas en este artículo- de centros de atención que consideren exclusivamente a este grupo etario. No obstante, su abordaje ha evolucionado en las últimas décadas, tomando perspectivas variadas dependiendo de la estructura de las políticas y los programas destinados a su atención (Cejas et al., 2008; Maceira, 2018).

Estos dispositivos consideran las particularidades de los/as adolescentes en función de dos miradas complementarias. En primer lugar, sus condiciones de vida presentes en relación con temáticas que las/os afectan particularmente, tales como adicciones, salud sexual, trastornos alimenticios y situaciones de violencia escolar o doméstica, que requieren atención concreta en consejería, prevención y cuidado. En segundo lugar, la inversión sanitaria en adolescencia genera externalidades en el mediano y largo plazo, en la medida que prevenir hábitos no-saludables y enfermedades que afecten su vida adulta, reduce riesgos de deterioro de la calidad de vida intertemporalmente, evitando asimismo mayores gastos directos de bolsillo del/la adolescente y su familia y del sistema de salud en el futuro. Este artículo, que forma parte de una investigación más amplia que incluye a las provincias de Jujuy, La Rioja, Misiones y Salta-, propone aportar a una agenda que aborde la temática desde diferentes perspectivas: los avances normativos orientados a establecer al/la adolescente como sujeto de derecho, la identificación de sus necesidades y la interacción con el círculo familiar y el sistema de salud, y la mirada de la política pública, comprendiendo y colaborando en el abordaje de tales necesidades. 
De Prácticas y discursos

Universidad Nacional del Nordeste

Centro de Estudios Sociales

Año 8, Número 11, 2019, Octubre

ISSN 2250-6942

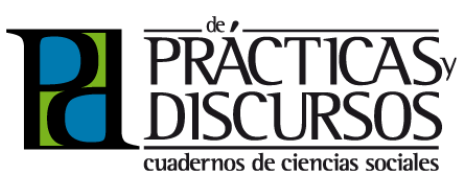

ABSTRACT Adolescence is a period of low clinical risk and incorporation of behavioral habits that will affect the transition to other life stages. In Argentina, progresses achieved in civil rights requires a proper correlation with accurate coverage of health needs. The interaction between the health and education systems and the role played by the family and the environment play a significant role in defining the burden of addictions, smoking habits, abuse of alcohol consumption, irresponsible sexual behaviors and passive and self-inflicted violence. This article uses quantitative-qualitative techniques to analyze the case of Chaco, including stakeholder analysis, normative review; interviews with civil society institutions; and the consolidation of a database collecting adolescents' perceptions about their health status and their ability to receive proper care from the health care system. It involves an anonymous multiple-choice survey completed by 800 students in twelve public high schools. These instruments measure key indicators, contrasting results across different geographical locations and genders. The study highlights the need for deeper coordination between public institutions and the families, to reduce the gap between adolescents' needs and adults' interpretation, and between the norm and its translation into a social protection system, facing a phenomenon that requires increasing attention.

Key words: adolescent, behavior, public policy.

\section{ANTECEDENTES Y MARCO CONCEPTUAI}

La bibliografía especifica sobre la temática de salud adolescente en el Chaco es escasa, y solo se cuenta con algunos informes de organismos internacionales y centros locales que han implementado proyectos o programas vinculados en la provincia (Unicef, 2013; Maceira et al., 2014; Garay et al., 2015). Estos artículos caracterizan a la adolescencia como una etapa de profundas transformaciones biológicas, psicológicas y sociales que tiene complejidades y particularidades atravesadas por el género, las condiciones materiales y culturales de vida, más allá de su condición de transición entre la infancia y la adultez (Garay et al., 2015). Asimismo, su abordaje desafía a los espacios existentes de participación (del sistema y del entorno familiar, comunitario y escolar) a fin de que los requerimientos de información y atención de los/as adolescentes se traduzcan en demandas y en un mejor acceso a la salud (Maceira, 2015).

En los últimos años han aparecido trabajos que de manera creciente describen la situación general de los adolescentes en el país, como el "Estado de la situación de la niñez y la adolescencia en Argentina", realizado por Unicef (2016), y la "Situación de las y los adolescentes en Argentina", del Ministerio de Salud de la Nación (2016); a ellos se agregan los que hacen hincapié en las temáticas de salud, como "El acceso a la salud de los y las Adolescentes en Argentina. Un análisis basado en evidencias", de Unicef (2017).

En el ámbito internacional, el estudio de la salud adolescente también ha sido un tema que concitó la mirada sanitaria durante los últimos años. Algunos trabajos que dan muestra de ello son Burt (1996) y los Informes de la Organización Mundial de la Salud en conjunto con Unicef (1999 y 2014) sobre Programación para la Salud de los Adolescentes, mencionando la relevancia de la oportunidad de invertir en salud en la segunda década de vida. Dentro de este marco se ubican asimismo el informe de Unicef (2011) sobre este particular y el documento regional sobre la estructura de los Programas de Salud en Niñez y Adolescencia en América Latina y el Caribe, como mecanismo para alcanzar la cobertura universal en salud (Maceira, 2015).

Estas contribuciones muestran su cercanía temporal (menos de diez años), reflejando la reciente visibilización del adolescente como sujeto particular. Dicha situación puede explicarse por los bajos niveles de mortalidad y morbilidad de esta franja etaria, lo 
que lleva a que no se consideren como un grupo de riesgo sanitario, salvo en los casos en que sus conductas sean vistas como problemáticas sociales de incidencia personal o comunitaria - el caso de las adicciones, la discriminación u otros modos de violencia. La articulación entre una etapa de profundos cambios y la falta de políticas públicas tendientes a atender sus necesidades, provocan deficiencias en lo que Maceira (2014) propone como componentes de la dimensión vertical en el estudio de los sistemas de salud: el aseguramiento, la gestión y la prestación de los servicios para adolescentes.

Nuestra investigación aporta a este debate, generando información que abona sobre los hallazgos de Maceira et al. (2014) acerca de los espacios vacantes de participación, entendiendo a la salud como un constructo social complejo, en el que intervienen diferentes actores. Sin embargo, hemos detectado que en las relaciones que constituyen el entramado territorial de la provincia, la participación del Estado -tanto nacional como subnacional- posee una centralidad particular, como garante del derecho al acceso a la salud, pero también como articulador entre lo público, las organizaciones de la sociedad civil y el entorno cercano del/la adolescente, especialmente cuando los vínculos entre ellos son insuficientes.

\section{PREGUNTAS DE INVESTIGACIÓN Y METODOLOGÍA}

Conceptualmente, el abordaje de la temática adolescente desde la política pública requiere comprender las necesidades objetivas -tanto clínicas como de formación psicosocial de este grupo etario-, como también el contexto económico y cultural -familiar y comunitario- en que ellos y ellas se desarrollan.

Sin embargo, una estrategia exitosa de abordaje debe también incorporar las interpretaciones (incluyendo preconceptos) de funcionarios/as y la sociedad en general acerca de tales necesidades y su abordaje, como también la traducción del/la propio/a adolescente de los mensajes recibidos por el mundo adulto y su lectura acerca de la validez de estas interpretaciones. De la confluencia de ambas se concreta el acceso eficaz y oportuno. La investigación llevada a cabo en la provincia del Chaco estuvo centrada en dos ejes: (i) analizar las caracteristicas y el funcionamiento del sistema público de salud provincial que presta servicios a la población adolescente; (ii) conocer la perspectiva de los/as usuarias/os adolescentes en relación con su estado de 
De Prácticas y discursos

Universidad Nacional del Nordeste

Centro de Estudios Sociales

Año 8, Número 11, 2019, Octubre

ISSN 2250-6942

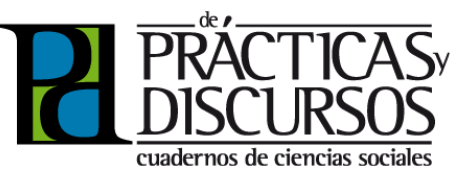

salud y las necesidades en esta materia, y la respuesta observada o percibida por parte del sistema público. El objetivo general perseguido por el proyecto fue la identificación de temáticas emergentes en la salud adolescente para el contexto chaqueño y su abordaje institucional. Los objetivos específicos surgidos del protocolo de trabajo se concentran en identificar prioridades de intervención asociadas con brechas entre regiones geográficas al interior de la provincia, entre necesidades de cuidado en la salud adolescente, identificando la potencial presencia de sesgos de género en tales necesidades.

El proceso de investigación involucró cuatro componentes: (i) una revisión y análisis de la literatura provincial, nacional e internacional sobre la temática de salud adolescente, (ii) un mapeo de actores provinciales vinculados con las estrategias públicas destinadas a adolescentes, mediante trece entrevistas semiestructuradas a los/as referentes de los Ministerios de Salud, Educación, Desarrollo Social, Derechos Humanos (Secretaría con rango ministerial) y el Instituto del Deporte asociados/as con el abordaje de la salud adolescente, (iii) la aplicación de una encuesta autoadministrada a ochocientos veintiséis adolescentes asistentes a catorce escuelas secundarias públicas de la provincia, seleccionadas como muestra para este estudio, y (iv) un grupo focal con referentes de asociaciones civiles que trabajan con adolescentes en situación de vulnerabilidad social, escolarizados y no escolarizados. Tanto las entrevistas en profundidad como las encuestas a estudiantes se llevaron a cabo entre septiembre y diciembre de 2016.

La selección de establecimientos tuvo una aproximación bietápica. En la primera, se escogió el grupo de los departamentos que formarian parte del estudio, que incluyó al distrito de la capital y de la segunda ciudad de la provincia, y una selección de tres departamentos que mostrara la diversidad socioeconómica del Chaco. Dentro de cada departamento se utilizó intencionalmente el mismo criterio de diversidad para escoger las escuelas públicas de la muestra.

En cada escuela se solicitó la respuesta a estudiantes del primer y último año, y los/as adolescentes contaron con cuestionarios de elección múltiple, contestados anónimamente y cuya participación fue de carácter optativo. El estudio contó con la aprobación del Comité de Ética del Hospital Pediátrico Dr. Castelán, como también con la autorización de la Dirección de Escuelas de 
la Provincia, dependiente del Ministerio de Educación, Cultura, Ciencia y Tecnología de la Provincia del Chaco, que facilitaron el trabajo en los establecimientos.

La metodología de análisis se basó -para el componente cualitativo- en la elección de palabras y términos clave alineados con los objetivos de este estudio y que posibilitaron el tabulado de las entrevistas en profundidad. Por otra parte, la carga de las encuestas de elección múltiple en hojas de cálculo fue el insumo que permitió generar una serie de cuadros y figuras sobre la cual se asienta el componente cuantitativo del documento. La Tabla 1 a continuación resume las principales características de la muestra.

\section{Descripción de la Muestra de Adolescentes Participantes, por departamento}

\begin{tabular}{lrrrrrr}
\hline $\begin{array}{c}\text { Departamentos } \\
\text { seleccionados }\end{array}$ & $\begin{array}{c}\text { \% población } \\
\text { total provincial }\end{array}$ & $\begin{array}{c}\text { \% Hogares } \\
\text { con NBI }\end{array}$ & $\begin{array}{c}\text { \% Población } \\
\text { rural }\end{array}$ & $\begin{array}{c}\text { N escuelas } \\
\text { encuestadas }\end{array}$ & $\begin{array}{c}\text { N estudiantes } \\
\text { encuestados }\end{array}$ & $\begin{array}{c}\text { \% total } \\
\text { estudiantes } \\
\text { encuestados }\end{array}$ \\
\hline San Fernando & 37,0 & 11,4 & 1,5 & 5 & 380 & 46,0 \\
Comandante Fernández & 9,2 & 16,4 & 14,2 & 4 & 103 & 12,5 \\
General Güemes & 6,4 & 35,6 & 34,9 & 2 & 179 & 21,7 \\
Libertador Gral. San Martín & 5,6 & 23,5 & 29,0 & 2 & 79 & 9,6 \\
Mayor Luis Fontana & 0,5 & 19,2 & 9,8 & 1 & 85 & 10,3 \\
Total & & & & $\mathbf{1 4}$ & $\mathbf{8 2 6}$ & $\mathbf{1 0 0 , 0}$ \\
\hline
\end{tabular}

FUENTE: Elaboración propia. Base CEDES-UNICEF.

\section{MARCO NORMATIVO}

Un modo de considerar un sistema de salud y su relación con la población de la que se ocupa es partiendo del marco normativo en el que se apoya, y a partir del cual se da reconocimiento formal a los derechos que declara garantizar.

Particularmente, el tratamiento de los derechos de niños, niñas y adolescentes ha experimentado internacionalmente un cambio radical de paradigma en las últimas dos décadas, del cual Argentina -y sus provincias- se ha hecho eco, adecuándose normativamente a él. Entre las más relevantes encontramos:

La Ley provincial N4276/96 de Creación del Programa de Educación para la Salud y Procreación Humana responsable, se propone poner a disposición de la población: educación, información, métodos y prestaciones de servicios que garanticen el derecho humano a decidir libre y responsablemente las pautas inherentes a su salud sexual, como así también y antes de la concepción de la vida; como también la Ley $N^{\circ} 7064$, vigente en la provincia 
De Prácticas y discursos

Universidad Nacional del Nordeste

Centro de Estudios Sociales

Año 8, Número 11, 2019, 0ctubre

ISSN 2250-6942

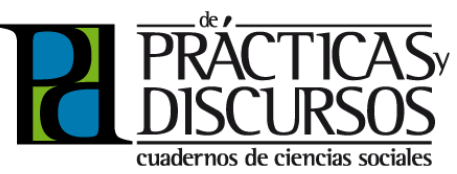

del Chaco desde octubre de 2012 que refiere a la aplicación de la Guía Técnica para la Atención Integral del Aborto no Punible. La Ley provincial N 7162/13 de Protección Integral de Derechos de Niñas, Niños y Adolescentes se sancionó en sintonía con la Ley nacional $N^{\circ} 26061$. Su objetivo es instituir en el territorio de la provincia, el Sistema de Promoción y Protección Integral de los Derechos de las Niñas, Niños y Adolescentes, garantizando el ejercicio y disfrute pleno, efectivo y permanente de los derechos consagrados en la Constitución nacional, en la Constitución provincial (1957-1994) y en tratados internacionales. Posee medidas de protección integral de los derechos.

La Ley provincial $N^{\circ} 7724 / 15$ crea el Instituto de la Mujer y la Familia (Imfa), buscando impulsar políticas con perspectiva de género y resguardar los derechos de grupos etarios vulnerables (incluidos los/as adolescentes) a través de la promoción de acciones que propicien y faciliten la plena incorporación de los mismos en la vida económica, cultural, política y social.

La Ley provincial N5811/06 sobre Educación Sexual Integral se enmarca en las leyes nacionales $N^{\circ} 23849, N^{\circ} 23179, N^{\circ} 26061$, $N^{\circ} 26150$, y en las leyes provinciales $N^{\circ} 4449$-General de Educación- y N 4.276 -Programa de Educación para la Salud y Procreación Humana Responsable y del concepto de sexualidad. Su objetivo es garantizar la enseñanza de los contenidos sobre la salud sexual, y alude y preserva los derechos sexuales y reproductivos desde una perspectiva de género, promoviendo el respeto a la diversidad y la no discriminación.

A través del Decreto provincial N 1934/16, Convenio Marco de Cooperación entre la Fundación del Hospital de Pediatría Profesor Dr. Juan P. Garrahan y el Ministerio de Salud Pública de la Provincia del Chaco, se busca promover y desarrollar en forma conjunta las acciones tendientes a disminuir la morbilidad y mortalidad materno infantil en la provincia del Chaco, creando redes y lazos recíprocos de colaboración en materia de actividad científica, técnica y capacitación en una concepción bio-psicosocial, abarcando los distintos niveles de la atención pediátrica con énfasis en la población materno infantil.

Maceira et al. (2018:37), en un estudio comparativo de cinco provincias (Salta, La Rioja, Chaco, Jujuy y Misiones), sostienen que en el ámbito normativo la pauta nacional no siempre precede a su tratamiento provincial, generando brechas de derechos entre jurisdicciones. En el caso chaqueño, sobre salud adolescen- 
De Prácticas y discursos

Universidad Nacional del Nordeste

Centro de Estudios Sociales

Año 8, Número 11, 2019, Octubre

ISSN 2250-6942
Salud adolescente en el chaco. Necesidades, actores y politicas

Adolescent health in Chaco. Needs, actors and policies

\footnotetext{
${ }^{7}$ Hay recurrencia en distinguir entre "consumo episódico excesivo" y alcoholismo. La problemática adolescente pasaría por el primero. Tan solo desde un centro especializado en la atención de adolescentes se mencionó tratar a jóvenes alcohólicos.
}

te, no se observa una adhesión sistemática de la provincia a la legislación nacional y en ocasiones se identifica liderazgo en el desarrollo de normativas. Tal es el caso de Salud Sexual y Reproductiva, donde la norma chaqueña se adelanta seis años a la nacional, o la Ley Provincial del Programa de Prevención y Control de Trastornos Alimentarios, que se sanciona un año antes que la equivalente ley nacional de 2008.

\section{MAPA DE ACTORES. NECESIDADES PERCIBIDAS, OBJETIVOS Y POLITICAS}

La presencia de normativa asociada con distintas necesidades de atención de la salud adolescente no establece una aplicación concreta de modo automático. Las políticas públicas implementadas desde el Ministerio de Salud y otras reparticiones del Estado provincial son aquellas que traducen la norma en acciones sobre la población objetivo. Estas acciones descansan -en teoríaen una estrategia de intervención que vincula necesidades con objetivos de política e intervenciones alineadas, aunque también se encuentran sujetas a las percepciones que los/as funcionarias/ os tienen sobre la realidad en la que actúan.

En esta sección analizaremos las percepciones de actores involucrados en políticas públicas que afectan a adolescentes, específicamente en temas referentes a salud; en torno al diseño, implementación y resultados de sus intervenciones.

Para ello se entrevistaron a trece actores clave en la temática, que cumplen roles en las áreas de Salud -responsables de direcciones, coordinadores de programas y efectores referentes de atención primaria-, Educación, Desarrollo Social y Derechos Humanos. Los ejes temáticos considerados fueron organizados en tres conjuntos: a. Problemáticas y necesidades claves de la población adolescente; $b$. Modos de abordaje, atención y contención: políticas para el acceso y la atención de la población adolescente y c. Barreras de acceso, brechas y falencias en demandaaccesibilidad del derecho a la salud de la población adolescente.

\subsection{PROBLEMÁTICAS Y NECESIDADES CLAVES DE LA POBLACIÓN ADOLESCENTE}

Hay concordancia entre los actores al definir a las problemáticas de salud en adolescentes como múltiples e interrelacionadas, 
De Prácticas y discursos

Universidad Nacional del Nordeste

Centro de Estudios Sociales

Año 8, Número 11, 2019, Octubre

ISSN 2250-6942

\footnotetext{
${ }^{8}$ En este punto no hay unanimidad en los/as entrevistadas/os. Algunos referentes (Salud y Desarrollo) sostienen esta postura; mientras que otro (Educación) lo desestima, sosteniendo que las escuelas cuentan con diferentes mecanismos para garantizar la permanencia de las estudiantes en estos casos.
}

destacándose: adicciones, consumo excesivo episódico de alcohol y maternidad no deseada.

Existe coincidencia en que el consumo excesivo episódico de alcohol" es "preocupante", por la masividad con que se lo percibe y los problemas que trae consigo -muertes y lesiones. El consumo de sustancias estupefacientes es elevado; principalmente marihuana y, en segundo lugar, residuos de otras drogas - paco.

El embarazo adolescente (EA), además de los múltiples riesgos que conlleva para la salud de la embarazada, deriva en situaciones no deseadas; reproducción de las condiciones de pobreza, aislamiento y deserción escolar ${ }^{8}$.

Los/as entrevistados/as coinciden en señalar que la condición material en donde tiene lugar el EA es fundamental, volviéndose un tema "más urgente" cuando la embarazada es una adolescente de bajos recursos económicos. Más allá de los riesgos propios del período de gestación, se menciona la falta de controles específicos asociados a la edad de la embarazada y la expresión vinculada a "la ausencia del Estado y el entorno a la hora de promover modelos de vida". Los entrevistados sostienen que, ante la falta de proyectos de realización, ligados al desarrollo laboral o al estudio, la maternidad emerge como "la única posibilidad de tener algo que les pertenezca".

Dentro del EA, las/os funcionarias/os de salud identifican como elemento crítico el alto índice del segundo embarazo adolescente. Si bien el primero es de por si un fenómeno preocupante, resulta un sintoma de alarma que se produzca el segundo "porque esa chica ya pasó por el sistema de salud", y este no pudo brindarle las herramientas necesarias para prevenirlo.

Otra problemática señalada, principalmente por referentes del sistema de salud, es el abuso sexual. Ellos/as lo asocian como un factor determinante de trastornos psicológicos: depresión y daños autoinfligidos, viendo en ellos el origen de los intentos de suicidios, adicciones, conductas violentas y dificultades de inserción institucional. Con respecto al suicidio, los actores coincidentemente sostienen que la tasa de suicidio adolescente es menor a la de otras provincias del país. Más allá de esta afirmación, las áreas de Salud y Derechos Humanos entienden que es un tema importante y que debe ser trabajado, ya que es expresión de otros problemas que afectan la calidad de vida de los adolescentes (tristeza, depresión, inseguridades, vulnerabilidad socioeconómica) o manifestación de situaciones psicológicas irresueltas -como la de abusos sexuales. 
De Prácticas y discursos

Universidad Nacional del Nordeste

Centro de Estudios Sociales

Año 8, Número 11, 2019, Octubre

ISSN 2250-6942
Salud adolescente en el chaco. Necesidades, actores y politicas

Adolescent health in Chaco. Needs, actors and policies

\footnotetext{
${ }^{9}$ iapa (Servicio Integral Amigable para Adolescentes) es un centro de salud público, que funciona en la localidad de R. Sáenz Peña.
}

Sin embargo, actores pertenecientes a otras áreas (Desarrollo Social, Educación y Deportes) manifiestan que, debido a la baja tasa de incidencia, no es un tema del cual el Estado deba ocuparse de manera prioritaria, en tanto: "Mueren más adolescentes en accidentes de tránsito, que por suicidios".

Emerge la necesidad de atender el pensamiento de suicidio ya que, aunque no resulte en la muerte puede traducirse en prácticas autodestructivas: autoflagelaciones -cortes, exceso o falta de abrigo deliberado, alteraciones en la alimentación-, comportamiento violento, etc. Al decir de un referente del sector, "estas conductas de riesgo afecta a la calidad de vida de los adolescentes".

Por último, se rescata dentro de las expresiones recibidas una distinción entre los problemas de salud de los/as adolescentes de la capital provincial con respecto a los que afectan a la población del interior: "mientras que para los de Resistencia se aplican estas temáticas (mencionadas anteriormente), a los del interior se le agregan enfermedades endémicas, como Chagas y Dengue", abriendo una dimensión geográfica sobre el peso de las enfermedades transmisibles en la adolescencia, que amerita de mayor profundidad.

\subsection{MODOS DE ABORDAJE, ATENCIÓN Y CONTENCIÓN: POLIITICAS PARA EL ACCESO Y LA ATENCIÓN A LA SALUD DE LA POBLACIÓN ADOLESCENTE}

Si bien los actores entrevistados consideran al adolescente como un sujeto con características particulares y, por lo tanto, demandante de una atención específica, diferenciada de los niños y adultos, no coinciden en el modo de plantear las intervenciones que se desprenden de esa consideración.

Por un lado, se considera que el ideal es que el sistema de salud responda a esta característica de la población creando secretarías, direcciones y programas cada vez más específicos, centrados en el adolescente. Esta es la postura de los referentes del área de salud, quienes manifestaron la necesidad de hospitales para adolescentes, diferentes y complementarios a los hospitales pediátricos y los hospitales para adultos. El Siapa ${ }^{9}$, si bien por sus dimensiones y recursos dista de ser un hospital, está creado y funciona bajo esta lógica. "Es como una salita para adolescentes", menciona su directora.

Otros actores sostienen que a pesar de que los adolescentes presentan características particulares, constituyen una etapa de transición, por lo que no amerita la creación de centros de salud 
De Prácticas y discursos

Universidad Nacional del Nordeste

Centro de Estudios Sociales

Año 8, Número 11, 2019, Octubre

ISSN 2250-6942

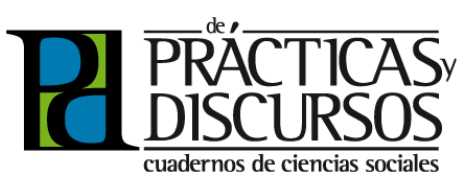

\begin{abstract}
${ }^{10}$ Según los Registros del Consultorio de Salud Reproductiva del Hospital Pediátrico Dr. A. Castelán, en los últimos años fue aumentando considerablemente el número de mujeres a quienes se les coloca DIU e Implantes Sub-dérmicos, y disminuyendo la edad en que lo solicitan.
\end{abstract}

11 Los talleres son mencionados por los referentes como los modos de intervención más exitosos, y en la mayoría de los casos, los/as entrevistados/as evidencian entusiasmo por los resultados obtenidos en los mismos. especializados; debiendo invertirse en programas que ataquen problemas específicos, sin énfasis en la cuestión etaria.

En el abordaje de la maternidad adolescente no deseada, hay consenso en el sector de salud en que la implementación del uso de anticonceptivos femeninos de larga duración es el modo más eficaz de tratarlo. Esto se basa en una creencia, sostenida en la experiencia de trabajo -sobre todo de los efectores de nivel primario- que los adolescentes "son inconstantes en sus hábitos $y$, por lo tanto, incapaces de sostener un método que demande una regularidad de ingesta y/o atención constante para su eficacia". ${ }^{10}$ Entre las intervenciones del área de salud, priman las charlas, los talleres con perspectivas lúdico-participativas para los adolescentes y los cursos destinados a efectores, junto a la provisión y/o gestión de recursos. Desde el Programa Provincial de Salud Integral en la Adolescencia también generan acompañamientos en procesos judiciales en casos de abusos.

Las charlas se hacen a través de los programas, el Ministerio de Desarrollo Social y el de Derechos Humanos, principalmente en escuelas, a partir de demandas concretas por parte de alguno de sus integrantes (centros de estudiantes, docentes, directivos, etc). También en centros comunitarios o espacios a los que sean convocados ${ }^{11}$.

El área de Derechos Humanos trabaja brindando charlas y talleres en donde se busca concientizar acerca de las consecuencias negativas del consumo excesivo. Asimismo, se brinda asesoramiento jurídico gratuito en conflictos en donde reconocen que se vulnere los derechos de los jóvenes.

Actualmente se intenta vincular las estrategias del sistema de salud con el de educación a través de un acuerdo interministerial que brinde un marco normativo a diferentes dispositivos que se desarrollan en el territorio, tales como las Asesorias en Salud y actividades de concientización acerca de la necesidad de cuidado, principalmente en torno a salud sexual y reproductiva.

\subsection{BARRERAS DE ACCESO, BRECHAS Y FALENCIAS EN DEMANDA - ACCESIBILIDAD DEL DERECHO A LA SALUD DE LA POBLACIÓN ADOLESCENTE}

Desde la perspectiva del sistema sanitario, los objetivos de las políticas provinciales están circunscriptos a áreas de salud específica: Maternidad, Salud Reproductiva y Salud Mental, por ejemplo. Perciben al adolescente como un sujeto con caracteristicas propias, pero no des- 
De Prácticas y discursos

Universidad Nacional del Nordeste

Centro de Estudios Sociales

Año 8, Número 11, 2019, Octubre

ISSN 2250-6942
Salud adolescente en el chaco. Necesidades, actores y politicas

Adolescent health in Chaco. Needs, actors and policies

\begin{abstract}
${ }^{12}$ Este aspecto es destacado por la mayoría de los entrevistados. Comentan que para acceder a un turno - por lo general se entregan diez por día para cada especialidad- los adolescentes deben concurrir con un adulto, a tempranas horas de la madrugada, y esperar la consulta que puede tener lugar ese mismo día o varios después.
\end{abstract}

\footnotetext{
${ }^{13}$ Según referentes del área de salud sexual y reproductiva, el sistema de registro actual no posibilita distinguir el tramo etario en las consultas de embarazadas en los Caps.
}

${ }^{14}$ Más allá de la capital provincial, solo se mencionan actividades en Mayor Fontana, Barranqueras -estas dos localidades conforman con Resistencia un único cordón urbano-, R. Sáenz Peña, J.J. Castelli y Villa Ángela. pliegan una estrategia destinada estrictamente a ellos/as, sino que las/ os contemplan en el marco de la necesidad de atención de la salud de la población en general. En este aspecto, el Programa de Salud Integral en la Adolescencia y el Siapa son los únicos diseñados exclusivamente para dar respuestas a la problemática de esta franja etaria. Junto al área de Educación sostienen que un impedimento para la atención es la falta de estructuras físicas y organizativas, que contemplen las necesidades de los adolescentes. Por ejemplo, la ausencia de dispositivos para un "control sano" y la dificultad para hacer consultas en los centros de salud pública'2.

Otra barrera a la hora de diseñar y aplicar políticas específicas para adolescentes es la falta de datos unificados y fiables, particularmente los provenientes del primer nivel de atención. Esta situación obstaculiza la capacidad de diagramar proyectos y evaluar su impacto ${ }^{13}$. Más allá de ello, emerge como una expresión difundida "la falta de recursos, tanto financieros como humanos, que impide extender los alcances de las actividades e intervenciones a todo el territorio provincial". Particularmente, desde el área de salud manifiestan la necesidad de medios de movilidad para cada programa, que permitan desempeñar sus funciones en el interior de la provincia, como el déficit de infraestructura en los lugares en donde desarrollan actividades. Este factor limita el alcance de las acciones llevadas a cabo, provocando que los cursos y capacitaciones, que son los instrumentos centrales para la "bajada" de los lineamientos de las políticas públicas hacia los efectores de salud se centralicen en la ciudad de Resistencia, dejando relegado al interior de la provincia ${ }^{14}$.

Desde el Ministerio de Desarrollo Social se hace hincapié en la falta de coordinación del sistema de salud con las actividades que realizan otros actores intervinientes en la problemática; "porque cada uno tienen sus prioridades", mencionan. Un caso evidente de ello es que todos los representantes de ministerios y organismos entrevistados manifiestan realizar algún tipo de actividad en las escuelas (talleres, charlas, torneos deportivos, etc.) pero cada uno desconoce las acciones de los otros.

\section{IDENTIFICACIÓN DE NECESIDADES POR PARTE DE LOS/AS ADOLESCENTES}

\subsection{LUGAR DE ATENCIÓN Y BARRERAS AL ACCESO}

La Figura 1 refleja para el total de los/as adolescentes encues- 
De Prácticas y discursos

Universidad Nacional del Nordeste

Centro de Estudios Sociales

Año 8, Número 11, 2019, 0ctubre

ISSN 2250-6942

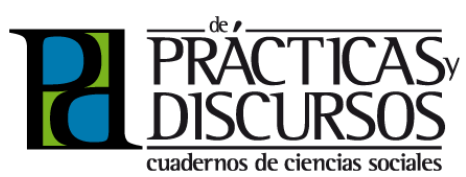

tadas/os, el lugar de concurrencia ante un evento de salud/enfermedad. Las opciones se abren entre el subsistema público en sus dos tipos generales de prestadores (hospitales y centros de atención primaria -Caps-), y la consulta privada, tanto mediante visita al médico como en sus variantes alternativas (farmacia como primera fuente de atención y curandero). Alternativamente, se consulta sobre la posibilidad de resolución en el hogar.

[Insertar Figura 1]

La atención en el hospital público y en el consultorio particular constituyen las principales fuentes de cuidado, distribuyéndose en el promedio provincial el 40,7\% y el 41,2\% del total respectivamente, exhibiendo la naturaleza mixta de la cobertura de salud del adolescente escolarizado en la escuela pública. Sin embargo, al observar al interior de la provincia, las/os adolescentes reflejan un sesgo hacia la institución pública, tal que, en $C$. Fernández, Mayor Fontana y General Güemes más del 55\% de las consultas se realiza en establecimientos dependientes de la autoridad provincial; en tanto, San Fernando y Libertador General San Martín evidencian la dirección opuesta. La relación prestador público/ privado se vuelca hacia la prestación no gubernamental en San Fernando, departamento con mayor índice de urbanidad y menor tasa de necesidades básicas insatisfechas, mientras que en el extremo opuesto se ubica Libertador San Martín, con el segundo indicador de NBI de la muestra.

Los centros de atención primaria de la salud no son considerados como un espacio de consulta usual, refiriendo un promedio de $10,6 \%$ del total de los/as respondientes, y extremos de $4 \%$ en General Güemes y 15,2\% en Mayor Fontana.

La asistencia a curanderos es mínima, con un valor superior del 2\% en Comandante Fernández, así como la consulta directa en farmacia, con un rango entre 1 y 3,6\%. La resolución en el hogar lleva un promedio relativamente reducido del 9\%, dispersándose entre el 6,3\% en Mayor Fontana y el 13,7\% en Libertador San Martín.

La Tabla 2 propone conocer cuáles fueron las dificultades de acceso a las instituciones de salud que expusieron las/os jóvenes, abierto por departamento. Más allá de las diferencias en los lugares de atención, en todos los casos los dos primeros motivos identificados como limitantes en el acceso son la falta de turnos y la demora en ser atendidos/as, superando ambos el 50\% de las opiniones adolescentes. Este dato es significativo, debido a que es coincidente con la visión de todos los actores involucrados 


\begin{abstract}
${ }^{15}$ Algunos espacios especializados en atención a adolescentes, como el Siapa (Servicio Integral Amigable para Adolescentes) en Sáenz Peña, han identificado esta barrera de acceso y abordado estrategias para su superación. Por ejemplo, el dispenser de preservativos y folleterias sobre salud sexual y reproductiva se encuentra en la entrada del edificio; un lugar seguro y de fácil acceso para las/os adolescentes, desde donde se los pueden retirar sin la mediación del agente sanitario.
\end{abstract}

(funcionarios/as, agentes sanitarios y sociedad civil), como también centra la limitante en el acceso a la dotación de recursos humanos, y no a la existencia de infraestructura.

En tercer lugar -con excepción de Güemes, donde figura en una cuarta colocación- surge una temática especifica en el debate sobre atención de adolescentes: la existencia de miedo o vergüenza que limita la asistencia o desanima la consulta. En el departamento con menor cantidad de opiniones al respecto, el 26,9\% (San Fernando) de la población involucrada en la encuesta considera este fenómeno como una limitación en el acceso, en tanto que en otros asciende a 34,8\% del total (Comandante Fernández), constituyendo un desafío en el diseño de una estrategia eficaz para este grupo etario ${ }^{15}$.

\begin{tabular}{lcccccc}
\multicolumn{6}{c}{ \% de estudiantes según dificultad de acceso a centro de salud } \\
\hline \multicolumn{1}{c}{ Municipio } & $\begin{array}{c}\text { San } \\
\text { Fernando }\end{array}$ & $\begin{array}{c}\text { Mayor Luis } \\
\text { Fontana }\end{array}$ & $\begin{array}{c}\text { Libertador } \\
\text { Gral. San } \\
\text { Martin }\end{array}$ & $\begin{array}{c}\text { General } \\
\text { Güemes }\end{array}$ & $\begin{array}{c}\text { Comandante } \\
\text { Fernández }\end{array}$ & Total \\
\hline Distancia & 13,5 & 15,1 & 13,8 & 30,5 & 25,0 & 18,9 \\
Mucho tiempo de espera/No lo ayudan & 64,1 & 56,2 & 72,3 & 58,3 & 53,3 & 61,3 \\
Falta de turnos/horarios & 56,1 & 47,9 & 53,8 & 61,6 & 48,9 & 55,3 \\
Falta de privacidad & 19,6 & 27,4 & 12,3 & 13,9 & 22,8 & 18,9 \\
Falta de información & 12,8 & 9,6 & 7,7 & 20,5 & 18,5 & 14,4 \\
Problemas financieros & 9,9 & 1,4 & 6,2 & 13,9 & 8,7 & 9,4 \\
Falta de atención adolescente & 12,8 & 12,3 & 13,8 & 12,6 & 8,7 & 12,3 \\
Falta de contención familiar & 6,1 & 4,1 & 3,1 & 2,0 & 3,3 & 4,3 \\
Miedo, vergüenza o no se anima & 26,9 & 28,8 & 32,3 & 28,5 & 34,8 & 29,0 \\
Falta de confianza & 10,6 & 11,0 & 6,2 & 12,6 & 14,1 & 11,1 \\
Sin dificultad & 16,8 & 13,6 & 13,7 & 6,4 & 10,0 & 13,1 \\
Otros & 4,5 & 8,2 & 4,6 & 1,3 & 5,4 & 4,3 \\
\hline
\end{tabular}

FUENTE: Elaboración propia en base a encuesta CEDESUNICEF.

Por otra parte, la barrera geográfica resulta significativa en General Güemes y Comandante Fernández, en tanto que la falta de privacidad es esgrimida como cuarta causa principal de limitación de acceso en San Fernando y Mayor Fontana con porcentajes en torno al 20\%.

La falta de contención familiar no aparece como una limitación, tampoco la carencia de información. En este último caso, la brecha identificada entre departamentos es extremadamente amplia: 20,5\% en General Güemes y 7,7\% en Libertador San Martín. Ante estos patrones de consulta y dificultades en el sistema de salud, la siguiente Tabla 3 pregunta sobre el entorno familiar de protección. Para ello se considera un grupo de cuatro variables tradicionales que fueron respondidas por el conjunto de adolescentes encuestadas/os: ¿Los padres escuchan? ¿Los/as consi- 
deran en la toma de decisiones del hogar? ¿Le hacen saber que pueden contar con ellos? ¿No están tan ocupados como para prestarles atención?

Para cada uno de los casos se implementó un indicador que es la tasa de respuesta de mujeres sobre la de los varones, al cual se le aplicó un test de significancia estadistica, a fin de identificar potenciales diferencias de género en la percepción de protección familiar.

Factores de Protección Familiar en Adolescentes (en \% del total)

\begin{tabular}{|c|c|c|c|c|c|c|c|c|}
\hline \multirow{3}{*}{ Municipio } & \multicolumn{6}{|c|}{ Sus padres: } & \multirow{2}{*}{\multicolumn{2}{|c|}{$\begin{array}{c}\text { No estan tan } \\
\text { ocupados para } \\
\text { prestarle atención }\end{array}$}} \\
\hline & \multicolumn{2}{|c|}{ Lo escuchan } & \multicolumn{2}{|c|}{$\begin{array}{c}\text { Lo consideran en la toma } \\
\text { de decisiones }\end{array}$} & \multicolumn{2}{|c|}{$\begin{array}{l}\text { Le hacen saber que puede } \\
\text { contar con ellos }\end{array}$} & & \\
\hline & $\begin{array}{l}\text { Total } \\
(\%)\end{array}$ & $\begin{array}{l}\text { Relación } \\
\text { M/V }\end{array}$ & $\begin{array}{c}\text { Total } \\
(\%)\end{array}$ & $\begin{array}{l}\text { Relación } \\
M / V\end{array}$ & $\begin{array}{l}\text { Total } \\
(\%)\end{array}$ & $\begin{array}{l}\text { Relación } \\
M / V\end{array}$ & $\begin{array}{l}\text { Total } \\
\text { (\%) }\end{array}$ & $\begin{array}{l}\text { Relación } \\
\text { M/V }\end{array}$ \\
\hline$\overline{\text { San Fernando }}$ & 70,94 & 0,83 & 55,07 & 0,89 & 85,55 & 0,98 & 82,07 & 0,95 \\
\hline Mayor Luis Fontana & 63,64 & 0,90 & 39,66 & 0,99 & 84,48 & 1,02 & 68,63 & 1,05 \\
\hline Libertador Gral. San Martín & 64,62 & 0,98 & 50,00 & 1,07 & 85,00 & 1,05 & 76,36 & 0,85 \\
\hline General Güemes & 63,06 & 0,89 & 43,96 & 0,98 & 83,33 & 0,96 & 87,18 & 1,00 \\
\hline Comandante Fernández & 60,53 & 0,96 & 45,45 & 1,07 & 82,35 & 1,15 & 72,22 & 0,96 \\
\hline Total & 67,12 & $0.88^{* *}$ & 50,48 & 0,95 & 84,73 & 1,01 & 80,07 & 0,95 \\
\hline
\end{tabular}

FUENTE: Elaboración propia en base a encuesta CEDESUNICEF.

Las respuestas dejan entrever un apoyo significativo de las familias, alrededor del $80 \%$ y del $84,73 \%$ en dos variables clave: recibir atención e informarles que pueden contar con ellos. En proporción menor, las/os adolescentes $(67,12 \%)$ se sienten escuchados, en tanto que más de la mitad de los/as jóvenes sienten que se los/as consideran en la toma de decisiones. Si bien existen diferencias entre departamentos, las mismas no parecen significativas. Se evidencia una brecha de "percepción de atención" entre géneros, siendo ella significativa contra las mujeres, particularmente en la capacidad de escucha familiar.

Este dato puede contrastarse con la percepción de los referentes de la sociedad civil, que concuerdan en sostener la ausencia del acompañamiento familiar en la cotidianeidad del/la adolescente como una de las principales barreras de acceso a la salud.

\subsection{PREVALENCIA DE FACTORES DE RIESGO ADOLESCENTE}

El estudio permite identificar los principales factores de riesgo sanitario de los/as adolescentes en la provincia, separando por unidad administrativa y por género. Las temáticas abordadas son: adicciones, conductas sexuales, sobrepeso, así como violencia de terceros y auto-infringida. Esta subsección y las siguientes exhiben 
los resultados de la investigación en cada uno de estos aspectos. Dentro de la temática de adiciones, la Tabla 4 analiza el consumo de tabaco, alcohol y drogas (al menos una vez en ambos casos), por jurisdicción, abierto por género. Se observa que el consumo de alcohol es más frecuente que el de drogas en una relación cercana en 2:1 en el promedio de la provincia, y de 4,8:1 con respecto al tabaco. Para tales valores promedio, las diferencias entre varones y mujeres son limitadas en alcohol y drogas, aunque cuenta con una incidencia masculina en tabaco en un 43\% mayor.

Incidencia de Adicciones en Adolescentes (en \% del total)

\begin{tabular}{lccc|ccc|ccc}
\hline \multirow{2}{*}{ Municipio } & \multicolumn{3}{c}{ Cosume tabaco } & \multicolumn{3}{c|}{$\begin{array}{c}\text { Cosumió alcohol } \\
\text { al menos una vez }\end{array}$} & \multicolumn{3}{c}{ Probó drogas en su vida } \\
\cline { 2 - 12 } & Total & Varón & Mujer & Total & Varón & Mujer & Total & Varón & Mujer \\
\hline San Fernando & 10,6 & 11,2 & 10,4 & 66,5 & 66,2 & 66,7 & 33,0 & 33,8 & 33,0 \\
Mayor Luis Fontana & 7,5 & 19,0 & 3,4 & 62,8 & 65,0 & 62,1 & 11,1 & 11,1 & 11,1 \\
Libertador Gral. San Martín & 15,6 & 15,6 & 15,6 & 75,6 & 71,9 & 78,3 & 44,4 & 35,3 & 52,6 \\
General Güemes & 14,9 & 19,2 & 11,8 & 65,8 & 65,4 & 67,2 & 26,6 & 25,9 & 28,6 \\
Comandante Fernández & 26,7 & 41,3 & 16,0 & 61,4 & 68,1 & 55,1 & 49,0 & 58,3 & 34,8 \\
Total & 13,7 & 18,6 & 10,8 & 66,2 & 66,8 & 66,1 & 33,5 & 35,2 & 32,4 \\
\hline
\end{tabular}

FUENTE: Elaboración propia en base a encuesta CEDESUNICEF.

No se verifica, sin embargo, un patrón homogéneo entre áreas geográficas. Tanto en tabaco como en drogas, el menor espacio de riesgo se encuentra en Mayor Fontana, particularmente en mujeres en el caso del tabaco, y una uniformidad cuatro veces menor al promedio provincial en consumo de drogas. En el extremo opuesto, Comandante Fernández ofrece la mayor exposición al alcohol y a las drogas, particularmente en el caso de los varones.

El patrón en el consumo de alcohol, si bien muestra que las diferencias existen y alcanzan el 18,7\% en promedios de género, son comparativamente más homogéneos, siendo Libertador General San Martín y Comandante Fernández los extremos.

La Tabla 5 refleja la realidad identificada en temáticas de conducta sexual, para lo cual se identifican tres trazadoras: presencia de relaciones sexuales (al menos una vez), edad de su primera relación y uso de preservativo en su último vínculo sexual.

Comparativamente, la edad de iniciación resulta similar entre géneros, alrededor de los quince años -marginalmente más temprano en los hombres ( 14 años y medio) que en las mujeres ( 15 años y tres meses). Aproximadamente la mitad de los/as adolescentes encuestadas/os tuvo relaciones sexuales en el rango 
etario estudiado. Siendo menor el número de respuestas positivas en Mayor Fontana (33,8\%) que en General Güemes (64,6\%), con una marcada diferencia entre varones y mujeres, cercana al 26\% para el promedio provincial. Libertador General San Martín es el único distrito donde la proporción de mujeres que declaran haber tenido relaciones sexuales es mayor a la de los varones.

Patrones Sexuales en Adolescencia (en \% del total)

\begin{tabular}{lccc|ccc|ccc}
\hline & \multicolumn{2}{c|}{$\begin{array}{c}\text { Tuvo relaciones sexuales } \\
\text { (\% estudiantes) }\end{array}$} & \multicolumn{2}{c|}{$\begin{array}{c}\text { Edad de la primera vez } \\
\text { (Años de edad promedio) }\end{array}$} & \multicolumn{2}{c}{$\begin{array}{c}\text { No se cuido en la última } \\
\text { relación sexual } \\
\text { (\%unicipio estudiantes) }\end{array}$} \\
\cline { 2 - 10 } & Total & Varón & Mujer & Total & Varón & Mujer & Total & Varón & Mujer \\
\hline San Fernando & 42,9 & 48,1 & 39,5 & 15,5 & 15,3 & 15,7 & 14,1 & 13,3 & 14,9 \\
Mayor Luis Fontana & 33,8 & 66,7 & 22,6 & 15,3 & 15,3 & 15,3 & 20,8 & 16,7 & 25,0 \\
Libertador Gral. San Martín & 57,3 & 53,3 & 60,0 & 15,2 & 14,7 & 15,4 & 21,4 & 13,3 & 25,9 \\
General Güemes & 64,6 & 69,6 & 57,4 & 14,5 & 14,1 & 15,0 & 22,6 & 34,9 & 5,4 \\
Comandante Fernández & 58,3 & $\mathbf{7 1 , 4}$ & $\mathbf{4 4 , 9}$ & 14,2 & 14,2 & 14,2 & 34,6 & 39,3 & 30,0 \\
Total & $\mathbf{4 9 , 6}$ & $\mathbf{5 8 , 2}$ & $\mathbf{4 2 , 9}$ & $\mathbf{1 5 , 0}$ & $\mathbf{1 4 , 7}$ & $\mathbf{1 5 , 3}$ & $\mathbf{2 0 , 5}$ & $\mathbf{2 4 , 1}$ & $\mathbf{1 6 , 9}$ \\
\hline
\end{tabular}

En otro orden, un quinto de los/as adolescentes declararon no haber usado protección en su última relación sexual, con valores extremos en Comandante Fernández $(34,6 \%)$, con una mayor toma de riesgo que en San Fernando $(14,1 \%)$. Dentro de este marco, los hombres $(24,1 \%)$ muestran menor cuidado que las mujeres (16,9\%), verificándose una asociación entre precocidad en la primera relación y falta de cuidado. Este dato también refuerza el hecho de que los indicadores más preocupantes -falta de cuidado- se encuentran en las localidades del interior provincial. Las tres jurisdicciones con mayor desprotección relativa -y mayor brecha entre géneros contra la población adolescente masculina- se asocia con altos niveles de pobreza.

Incidencia de Sobrepeso (en \% del total)

\begin{tabular}{lrcc}
\hline \multicolumn{1}{c}{ Municipio } & \multicolumn{3}{c}{ Sobrepeso } \\
\cline { 2 - 4 } \multicolumn{1}{c}{ Total } & Varón & Mujer \\
\hline San Fernando & $11,3 \%$ & $13,4 \%$ & $10,3 \%$ \\
Mayor Luis Fontana & $12,7 \%$ & $20,0 \%$ & $10,0 \%$ \\
Libertador Gral. San Martín & $11,7 \%$ & $0,0 \%$ & $19,4 \%$ \\
General Güemes & $15,0 \%$ & $12,7 \%$ & $19,3 \%$ \\
Comandante Fernández & $11,6 \%$ & $12,1 \%$ & $12,1 \%$ \\
Total & $\mathbf{1 2 , 2 \%}$ & $\mathbf{1 2 , 1 \%}$ & $\mathbf{1 2 , 7 \%}$ \\
\hline
\end{tabular}

FUENTE: Elaboración propia en base a encuesta CEDESUNICEF. 
De Prácticas y discursos

Universidad Nacional del Nordeste

Centro de Estudios Sociales

Año 8, Número 11, 2019, Octubre

ISSN 2250-6942
Salud adolescente en el chaco. Necesidades, actores y politicas

Adolescent health in Chaco. Needs, actors and policies
${ }^{16}$ En este sentido se apela a diferentes recursos como, por ejemplo, obras de teatro, de títeres, etc., para que los adolescentes se sientan "útiles y pertenecientes a algo".

${ }^{17}$ Otras actividades mencionadas son las relacionadas con el cuidado del ambiente: plantada de árboles, reciclado de botellas, etc.

Finalmente, la incidencia de sobrepeso en la provincia es del $12,2 \%$, con mayor prevalencia relativa entre las mujeres. Las jurisdicciones presentan perfiles similares en el total; sin embargo, muestran marcadas diferencias en el contraste entre géneros en Mayor Fontana donde se declaran diferencias de 20\% en varones y $10 \%$ en mujeres; al igual que General San Martín, que presenta valores de $0 \%$ para varones y $19,4 \%$ para mujeres. En contraste a estos últimos, los municipios de San Fernando (13,4\%-10,3\%) y Comandante Fernández $(12,1 \%-12,1 \%)$ muestran paridad en la desagregación por géneros.

\subsection{ACCIONES DE VIOLENCIA: SITUACIONES DE PELEAS, AGRESIONES Y LESIONES}

En lo referido a acciones de violencia, se preguntó a las/os adolescentes sobre su participación en situaciones de peleas, agresiones y lesiones, distinguiendo por sexo y localidad. Las peleas se constituyen en el fenómeno de violencia más frecuente en este tipo de situaciones en la provincia (con excepción de Mayor Fontana). El promedio de 20\% de adolescentes participantes para la jurisdicción exceptúa a Comandante Fernández, donde las mismas involucran a la mitad de las/os jóvenes encuestados. Los hombres son más propensos a exponerse a estos hechos, superando en general a las mujeres, donde el fenómeno de lesiones tiene mayor protagonismo.

Incidencia de Acciones de Violencia (en \% de adolescentes)

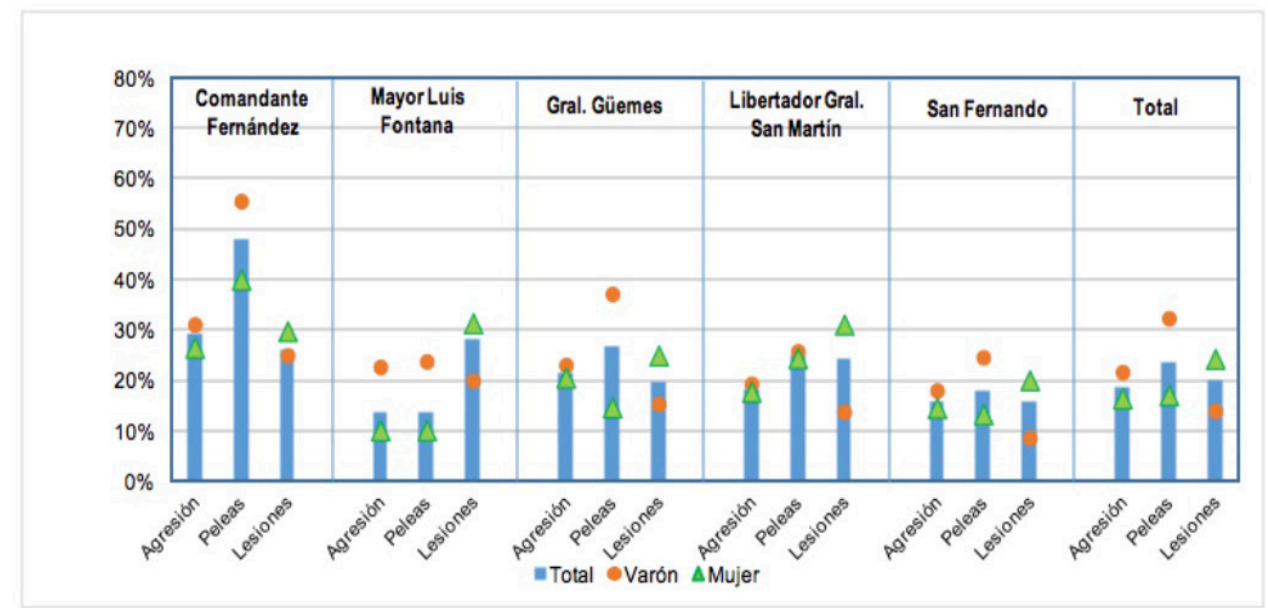

FUENTE: Elaboración propia en base a encuesta CEDESUNICEF. 
Por último, la Tabla 7 muestra las respuestas recogidas sobre pensamiento e intento de suicidio, por locación y por género. Los valores son significativamente altos: $28,5 \%$ del total provincial tuvo pensamientos asociados al suicidio, y 5,5\% lo ha intentado. Del conjunto chaqueño, Mayor Fontana $(8,6 \%)$ y Comandante Fernández $(7,4 \%)$ son las de más alta incidencia promedio en el intento de suicidio. Siendo General Güemes $(3,7 \%)$ la de menor valor para este índice. Para todos los casos, la temática cuenta con un fuerte sesgo femenino, con una relación de 2 a 1 en pensamiento de suicidio y cercana a 4,5 veces mayor en el intento de hacerlo.

\section{Incidencia de suicidio y pensamiento de suicido (en \% del total)}

\begin{tabular}{lccc|ccc}
\hline \multirow{2}{*}{ Municipio } & \multicolumn{3}{c|}{ Pensó en suicidarse } & \multicolumn{3}{c}{ Pensó e intento suicidarse } \\
\cline { 2 - 7 } & Total & Varón & Mujer & Total & Varón & Mujer \\
\hline San Fernando & 30,3 & 17,5 & 37,1 & 5,0 & 1,6 & 7,0 \\
Mayor Luis Fontana & 30,0 & 10,0 & 38,0 & 8,6 & 0,0 & 12,0 \\
Libertador Gral. San Martín & 30,7 & 17,2 & 39,1 & 5,3 & 3,4 & 6,5 \\
General Güemes & 24,3 & 15,6 & 34,4 & 3,7 & 1,6 & 6,3 \\
Comandante Fernández & 25,3 & 20,9 & 29,8 & 7,4 & 2,3 & 12,8 \\
Total & $\mathbf{2 8 , 5}$ & 17,0 & $\mathbf{3 6 , 2}$ & $\mathbf{5 , 5}$ & $\mathbf{1 , 8}$ & $\mathbf{8 , 0}$ \\
\hline
\end{tabular}

FUENTE: Elaboración propia en base a encuesta CEDESUNICEF.

\section{SOCIEDAD CIVIL: INICIATIVAS Y MODOS \\ DE ARTICULACIÓN CON EL SECTOR PÚBLICO EN EL TRATAMIENTO DE LA CUESTIÓN ADOLESCENTE}

La articulación entre la sociedad civil y el Estado es siempre objeto de análisis, particularmente en temáticas asociadas con políticas públicas. En este caso en particular, el interés en involucrar la voz de las OSC tiene una motivación adicional, buscar capturar indirectamente las opiniones y percepciones de la adolescencia no-escolarizada.

En la provincia del Chaco existen escasas OSC destinadas exclusivamente a adolescentes. Sin embargo, entre las que orientan sus acciones a población general, consideran a este grupo entre los más vulnerables. De las cuatro entrevistadas, dos se desempeñan como centro de enseñanza; una de ellas, dependiente de una entidad religiosa, la segunda es una escuela de gestión social, institución pública autónoma. Una de las dos OSC restantes se define como una institución de 
investigación, y la otra está asociada a un partido político.

Los problemas reconocidos coinciden con los mencionados en las entrevistas a actores clave, con un mayor énfasis en el consumo de sustancias y salud reproductiva. Las acciones llevadas a cabo incluyen talleres y charlas sobre problemáticas detectadas en el territorio: consumo de alcohol y drogas, salud reproductiva, enfermedades de transmisión sexual y violencia. En el caso de los/as adolescentes, se orientan a acciones de contención para "mantenerlos ocupados" y "fuera de las calles"16; se destaca la organización de eventos deportivos, los cuales son considerados como herramientas clave en esta dirección ${ }^{17}$.

Con respecto a la población no escolarizada, los casos de contacto de las OSC se dan a raíz de las problemáticas con más incidencia en el terreno de acción, como ser adicciones, salud reproductiva y enfermedades de transmisión sexual. Las OSC no identifican diferencias entre las necesidades reconocidas en la población adolescente escolarizada y no escolarizada, sino que las agrupan como problemáticas generales de este sector, sin que la escolarización suponga un elemento distintivo. A su vez, afirman que los adolescentes en situación de no escolaridad son escasos, y hacen hincapié en situaciones intermedias, o de escolarización precaria: adolescentes que concurren eventualmente a una institución escolar, pero que no logran tener una regularidad en ella, que están "siempre en el límite de dejar la escuela", por lo que consideran poco efectivo asociar escolaridad con matriculación en una institución.

En cuanto a las dificultades de acceso a la salud, la falta de recursos económicos representa el motivo fundamental, sumado a la escasa contención familiar o ausencia de una cultura del cuidado de la salud: "la barrera de ellos me parece que constituye la problemática económica [...] Tienen otras prioridades, por ejemplo, la comida, la vivienda, después la seguridad y la salud va quedando rezagada", sostiene una de las entrevistadas.

Es notable esta coincidencia entre los participantes en marcar la falta de recursos económicos como principal barrera de acceso, porque difiere de lo que había surgido en las entrevistas a actores clave. Estos últimos, en su mayoría funcionarios estatales, vinculaban el problema a una característica de la constitución (psicológica) propia del adolescente. Aunque, en algunos casos, se reconocía como un determinante la organización del sistema de salud, como ser, y aqui hay coincidencias, la dificultad para conseguir turnos. 
De Prácticas y discursos

Universidad Nacional del Nordeste

Centro de Estudios Sociales

Año 8, Número 11, 2019, Octubre

ISSN 2250-6942

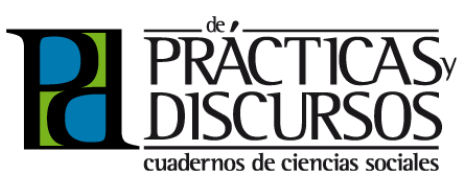

Se evidencia una doble carencia en los adolescentes pobres, que determina una barrera central para el cuidado de su salud. Por un lado, falta de recursos económicos, que relega la atención en el orden de prioridades y, por el otro, falta de lo que puede considerarse como un capital simbólico (Bourdieu y Wacquant, 2005), que se traduciría como ausencia de una cultura del cuidado, de conocimientos de cómo funciona el sistema de atención y de redes que les permitan accionar -atenderse- a pesar de las deficiencias de ese sistema. Las OSC pretenden subsanar esta falta, por ejemplo, a partir de la gestión de turnos o de la organización de jornadas sanitarias, en donde los profesionales concurren a los centros de enseñanza para algún tipo de control de salud.

\section{CONCLUSIONES}

El artículo busca poner en evidencia los elementos que componen una tensión entre la necesidad de acceder al servicio de salud por parte de los adolescentes y el modo en que este derecho es formal y efectivamente reconocido en la práctica en la provincia del Chaco. Las problemáticas de salud que atraviesan a la población adolescente en la provincia pueden ser resumidas en cuatro principales: adicciones a sustancias estupefacientes, principalmente a marihuana y residuos; consumo excesivo episódico de alcohol; embarazos no deseados y las derivadas de abusos sexuales.

Los funcionarios del área salud correlacionan el problema de las adicciones a la aparición de otras temáticas como desnutrición y muertes por causas externas, en tanto desde el sector educativo, lo vinculan a la deserción y a la violencia escolar. Por su parte, los representantes de las OSC hacen hincapié en las condiciones socio-culturales en que los adolescentes inician el consumo: falta de contención familiar, necesidades insatisfechas, falta de espacios y actividades que tiendan al fortalecimiento del sentido de pertenencia a una comunidad (familiar, barrial, política).

Las instituciones provinciales dan respuesta a estas problemáticas a partir de la implementación de lineamientos de políticas de salud pública nacionales, a través de Programas como el de Salud Reproductiva o el Programa Provincial de Salud Integral de Adolescentes, generando a partir de ellos espacios de participación y coordinación con otras áreas de gobierno. La provisión y la gestión de recursos es una parte importante de la labor de las Secretarias y las Direcciones. 
La ausencia de infraestructura y medios de movilidad produce escasa distribución de insumos, dificultades en la comunicación y formación de los agentes de salud, y una centralización de las acciones en la capital provincial. Esta situación genera una brecha entre lo que formalmente se garantiza y lo que efectivamente acontece en el territorio.

Otros factores que dificultan la promoción del derecho a la salud en este grupo etario es la falta de coordinación entre las diferentes áreas del gobierno que trabajan simultáneamente con adolescentes, tanto al interior del Ministerio de Salud como de este con sus pares de Educación y Desarrollo Social. En este mismo sentido, también existe precariedad en los datos disponibles para el diseño de las acciones.

La estructura organizativa de la atención en centros de salud y hospitales, como también en el sector privado en ocasiones, se presenta como una barrera importante para la concurrencia del adolescente al centro de salud. La dificultad de obtener turnos para las consultas y la demora en ser atendidos aparecen como las razones más recurrentes que impiden el control sanitario.

Algunas discrepancias entre los datos obtenidos en las encuestas a estudiantes con lo mencionado por referentes de las OSC, habilita a suponer que existen otros factores que inciden en sus problemáticas y necesidades. En las entrevistas a estudiantes se muestra que el 80\% considera tener contención familiar, mientras que, según las OSC, hay ausencia de lazos familiares fuertes. Si consideramos que las encuestas fueron aplicadas a estudiantes de escuelas secundarias formales, y los miembros de las OSC trabajan con adolescentes en programas escolares especiales, no estaríamos ante una contradicción en los datos, sino ante una distinción de situaciones/necesidades/problemáticas diferenciadas según el grado de vulnerabilidad socioeconómica que posea el adolescente.

Esto último nos permite suponer que es existe la necesidad de cuestionar la construcción del sujeto (Foucault, 2012) "adolescente" como algo homogéneo y determinado estrictamente por un factor etario. Las políticas públicas de salud no deben quedar circunscriptas a una serie de caracteristicas predefinidas, consecuencia de perspectivas sesgadas fundamentalmente por lo biológico y las particularidades socio-culturales (Rozé et al., 1999). Se hace necesario profundizar una discusión territorial que contemple las singularidades de los actores involucrados, a fin de garantizar efectivamente el acceso a la salud desde una perspectiva inclusiva. 


\section{BIBLIOGRAFIA}

ADLER-LOMNITZ, L. (2012). Redes sociales, cultura y poder: ensayos de antropología latinoamericana. México DF: Facultad Latinoamericana de Ciencias Sociales.

BURT, M. (1996). ¿Por qué debemos invertir en el adolescente? OPS. Washington D.C.

CEJAS, C.; OLAVIAGA, S. Y STECHINA M. (2008). ¿Qué piensan los jóvenes? Estudio comparado sobre la percepción de los jóvenes acerca de la salud, las instituciones y sus derechos en cinco municipios. Documento de Trabajo, 20, Cippec, Buenos Aires, noviembre.

BOURDIEU, P. (2008). Capital Cultural, Escuela y Espacio Social. Buenos Aires: Siglo XXI.

BOURDIEU, P. Y WACOUANT, L. (2005). Una invitación a la sociología reflexiva. Buenos Aires: Siglo XXI.

FOUCAULT, M. (2012). Nacimiento de la Biopolítica. Buenos Aires: FCE.

GARAY, E.; TEDESCHI, V. Y AULICINO, C. (2015). Las políticas de adolescencia en la provincia de Chaco. Documento de trabajo, 138, Cippec, Buenos Aires.

GOVEA BASCH, J. Y DE PAULA, M. (2016). Indicadores para el seguimiento de la situación de salud de la población adolescente. Unicef Argentina. Inédito.

MACEIRA, D. (2014). "Cuadrantes de Análisis en los Sistemas de Salud de América Latina", Documento de Trabajo, 122, Área de Economía, Centro de Estudios de Estado y Sociedad (Cedes) / Unicef Lacro. Diciembre.

(2015). Estructura de los Programas de Salud en Niñez y Adolescencia en América Latina y el Caribe. Espacios Institucionales para alcanzar la Cobertura Universal en Salud. Documento de Trabajo, 123, Área de Economía, Centro de Estudios de Estado y Sociedad (Cedes)/Unicef Lacro. Mayo. 
(2018). Programas de salud adolescente en Argentina. Objetivos, prioridades y estructura organizacional. Documento de Trabajo, 134. Buenos Aires: Cedes.

MACEIRA, D.; HASICIC, C.; CARLINO, M.; IBARRA, M.; MARCOS, P.; NAVARRO, D. Y SADIR, F. (2018). Marco normativo, federalismo y salud adolescente. El caso de cinco provincias del norte argentino. Documento de Trabajo, 135. Buenos Aires: Cedes.

ORGANIZACIÓN MUNDIAL DE LA SALUD (1999). "Informe de un grupo de estudio OMS/Funap/Unicef sobre Programación para la Salud de los Adolescentes". Serie de Informes Técnicos, 886. OMS. Ginebra.

(2014). Salud para los adolescentes del mundo. Una segunda oportunidad en la segunda década. Recuperado de http://apps.who.int/adolescent/seconddecade/files/WHO_FWC_ MCA_14.05_spa.pdf?ua=1\&ua=1

PROGRAMA NACIONAL DE SALUD INTEGRAL EN LA ADOLESCENCIA, MSAL Y UNICEF (2016). Situación de las y los adolescentes en Argentina. Buenos Aires: PNSIA-Unicef.

REPETTO, F., DÍAZ LANGOU, G. Y FLORITO, J. (2016). Políticas provinciales para las adolescencias. ¿Dónde estamos y hacia dónde vamos? Buenos Aires: Unicef-Cippec.

ROZÉ, J.; PRATESI, A. Y BENITEZ, A. (1999). Trabajo, moral y disciplina en los chicos de la calle. Resistencia: Espacio editorial. Unicef (2011). Estado mundial de la infancia 2011. La Adolescencia, una época de oportunidades. Nueva York.

UNICEF ARGENTINA (2016). Estado de la situación de la niñez y la adolescencia en Argentina. Buenos Aires: Unicef Argentina.

(2017). El acceso a la salud de los y las Adolescentes en Argentina. Un análisis basado en evidencias. . Buenos Aries: Unicef Argentina.

(2018). www.unicef.org/argentina. [Obtenido de] https://www.unicef.org/argentina/que-hace-unicef/salud/ asesor\%C3\%ADas-de-salud-integral-en-escuelas-secundarias 